\title{
Plaquette valence bond theory of high-temperature superconductivity
}

\author{
Malte Harland \\ Institut für Theoretische Physik, Universität Hamburg, Jungiusstraße 9, 20355 Hamburg, Germany \\ Mikhail I. Katsnelson \\ Radboud University, Institute for Molecules and Materials, Heyendaalseweg 135, 6525AJ Nijmegen, The Netherlands \\ Alexander I. Lichtenstein \\ The Hamburg Centre for Ultrafast Imaging, Luruper Chaussee 149, Hamburg 22761, Germany \\ and Institut für Theoretische Physik, Universität Hamburg, Jungiusstraße 9, 20355 Hamburg, Germany \\ (Received 10 April 2016; revised manuscript received 26 July 2016; published 19 September 2016)

\begin{abstract}
We present a strong-coupling approach to the theory of high-temperature superconductivity based on the observation of a quantum critical point in the plaquette within the $t, t^{\prime}$ Hubbard model. The crossing of ground-state energies in the $N=2-4$ sectors occurs for parameters close to the optimal doping. The theory predicts the maximum of the $d_{x^{2}-y^{2}}$-wave order parameter at the border between localized and itinerant electron behaviors and gives a natural explanation for the pseudogap formation via the soft-fermion mode related to local singlet states of the plaquette in the environment. Our approach follows the general line of resonating valence-bond theory stressing a crucial role of singlets in the physics of high- $T_{c}$ superconductors but focuses on the formation of local singlets, similar to phenomena observed in frustrated one-dimensional quantum spin models.
\end{abstract}

DOI: 10.1103/PhysRevB.94.125133

\section{INTRODUCTION}

After the 30-year history of extremely intensive experimental [1-4] and theoretical [5-10] studies of the high-temperature superconductivity (HTSC) in copper oxides we are still far from understanding the basic mechanism of this fascinating phenomenon. Taking into account the enormous number of researchers involved in this field, one can assume that almost all possible ideas were expressed and that the main problem is just to select the basic simple concepts from the pile of available theoretical results. The most ambitious attempt was made by Anderson who emphasized with his resonating valence-bond (RVB) theory the crucial importance of strong electron correlations, the tendency for singlet spin state formation, and the non-Fermi-liquid character of the normal phase [6]. Unfortunately, details of his original approach, such as suppression of interlayer hopping in the normal phase as the main factor of superconductivity, seem to contradict experimental data [11]. The latest version of the RVB theory is presented in Ref. [12]. We believe that the main assumption of the strongly correlated limit as the base of understanding the high-temperature superconductivity is correct as well as emphasizing a crucial role of spin singlet states but important details were missing. Below we present arguments for the thesis that the minimal object of HTSC theory is the plaquette in the so-called effective $t, t^{\prime}$ Hubbard model $[13,14]$ rather than the conventional atomic limit typical for the theory of Mott insulators [6,9]. The best practical realization of this atomic-based theory is the dynamical mean-field theory (DMFT) [15]. The obvious minimal generalization in the case of $d_{x^{2}-y^{2}}$-wave pairing is a cluster DMFT (CDMFT) scheme $[16,17]$.

Since the first plaquette CDMFT calculation of $d_{x^{2}-y^{2}}$ wave superconducting order together with antiferromagnetic fluctuations [16], there have been many calculations for different cluster sizes and geometries based on continuous-time quantum Monte Carlo (CTQMC) or exact diagonalization solvers [17-35]. Unfortunately, the basic qualitative feature of the many-body states in the plaquette were hidden in the computational details. The main aim of this paper is to present a simple and transparent strong-coupling theory of the $d_{x^{2}-y^{2}}$-wave superconductivity, i.e., a minimal consistent many-body model, based on the plaquette energy spectrum peculiarity, namely, the "quantum critical point" that merges two singlets and two doublets. These states of the doped plaquette are different from those discussed in the resonating valence-bond theory $[6,12]$. The main point is that the quantum critical point discussed here is related to the formation of local valence bonds in the frustrated quantum spin model [36]. Therefore, the optimal superconducting states are located on the border between localized and delocalized resonating plaquette valence bonds. Here we follow a bottom-up approach starting with an isolated plaquette and building stepwise a more complex environment.

An important theoretical problem is to find a minimal and generic electronic-structure model of cuprate superconductors. From band-structure calculations $[13,14]$ we can safely reduce it to an effective one-band model with nearest- and nextnearest-neighbor hopping. We use a standard parametrization of the tight-binding model for $\mathrm{YBa}_{2} \mathrm{Cu}_{3} \mathrm{O}_{7}[13,14]$ with the next-nearest-neighbor hopping: $t^{\prime} / t=-0.3$ and $t$ as units of our energies. The local Hubbard interaction parameter $U$ is on the order of the bandwidth $W=8 t$. Then, the $t, t^{\prime}$ Hubbard model on the square lattice reads

$$
H=-\sum_{i j} t_{i j} c_{i \sigma}^{\dagger} c_{j \sigma}+\sum_{i} U n_{i \uparrow} n_{i \downarrow},
$$

where $t_{i j}$ is an effective hopping and $U$ is the local Coulomb interaction. The operators $c_{i \sigma}^{\dagger}, c_{i \sigma}$ create and annihilate fermions, respectively, at site $i$ with spin $\sigma=\uparrow(+), \downarrow(-)$, and the 


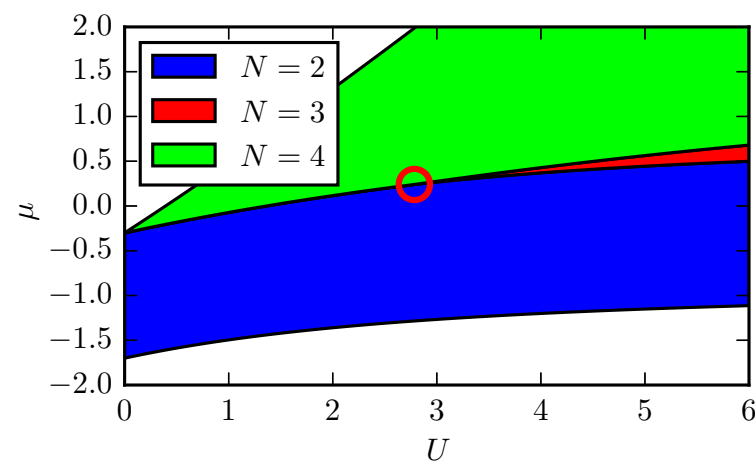

FIG. 1. Zero-temperature phase diagram of the isolated plaquette as a function of the Hubbard $U$ and chemical potential $\mu$ in the proximity of the quantum critical point (circle) for $t^{\prime} / t=-0.3$.

occupation operator is $n_{i \sigma}=c_{i \sigma}^{\dagger} c_{i \sigma}$; furthermore we will use $t$ as the energy unit.

\section{ISOLATED PLAQUETTE}

We start the discussion with electronic states in the isolated Hubbard plaquette. The optimal doping for high-temperature superconductivity is on the order of $15 \%$ of holes per site for almost all cuprate materials. This gives us an average number of electrons per site of 0.85 and results in 3.4 fermions per four-site plaquette in the crystal. We argue that this is related to three-electron states of the isolated plaquette since particle-hole asymmetry introduced by the next-nearestneighbor hopping $t^{\prime}$ with moderate values of $U$ and certain fixed chemical potentials $(\mu)$ result in an occupation per plaquette of the crystal, that is very close to the optimal value of 3.4 electrons.

The Hamiltonian of the isolated plaquette reads

$$
\begin{aligned}
H_{p} & =\sum_{(i, j)=1 \ldots 4} h_{i j}^{0} c_{i \sigma}^{\dagger} c_{j \sigma}+\sum_{i=1 \cdots 4} U n_{i \uparrow} n_{i \downarrow}, \\
-\hat{h}_{0} & =\left(\begin{array}{cccc}
\mu & t & t^{\prime} & t \\
t & \mu & t & t^{\prime} \\
t^{\prime} & t & \mu & t \\
t & t^{\prime} & t & \mu
\end{array}\right) .
\end{aligned}
$$

We include the chemical potential in the diagonal part of $h_{i j}^{0}$. The energy spectrum of the isolated plaquette near the three-electron filling is very unusual. We present in Fig. 1 regions in the $U-\mu$ space, whose ground states have an occupation of three plus-minus one electrons. The one-electron spectrum possesses four states with the energies: $\pm 2 t-t^{\prime}-\mu$ and double-degenerate $t^{\prime}-\mu$. At zero interaction $U=0$, there is no stable ground state with three electrons in the sense that one can add or remove one electron without changing the thermodynamic potential. Starting from some critical interaction strength $U \approx 3$ there is a small region in that the plaquette ground state with $N=3$ electrons is separated by energy gaps from the states with $N=2$ and $N=4$, see Fig. 1. Importantly, this $N=3$ ground state is fourfold degenerate consisting of two doublets in the sectors $\left(2_{\uparrow}, 1_{\downarrow}\right)$ and $\left(1_{\uparrow}, 2_{\downarrow}\right)$, which we label $X$ and $Y$, according to their symmetry. Moreover, there is a critical point (circle in Fig. 1) where all three sectors with two through four electrons have the same ground-state energy and form a sixfold degenerate ground-state multiplet consisting of two singlets of the sectors $\left(1_{\uparrow}, 1_{\downarrow}\right)$ and $\left(2_{\uparrow}, 2_{\downarrow}\right)$ together with two doublets of the three-electron sectors. This critical point has the coordinates $U=2.78, \mu=0.24$ for the standard value of $t^{\prime} / t=-0.3$. We think that this critical point of the plaquette has crucial importance for the physics of the strong-coupling $d_{x^{2}-y^{2}}$-wave superconductivity. The importance of these three many-body states of the plaquette CDMFT has been discussed first for the $t$ - $J$ model [19]. In that case an additional triplet state in the $N=4$ sector appeared without formation of the single quantum critical point. The crossing of different many-body states has been investigated in the valence-bond DMFT [20], the checkerbord Hubbard model [37], and in the plaquette CDMFT $[27,33]$. The idea of a quantum critical point and nematicity has also been discussed in Refs. [23,31,32]. Here, we demonstrate via a bottom-up approach that this is the key ingredient of a consistent minimal picture of HTSC.

If we approach this critical point from the region with the $N=3$ ground state, then the one-electron density of states (DOS) at the Fermi energy diverges for both electron and hole sides due to transitions from the fourfold degenerate $N=3$ ground state to singlets of $N=2$ (hole side) and $N=4$ (electron side) with zero excitation energy. The corresponding spectral weights with normalization of the $\delta$ functions are equal to 0.46 and 0.23 for the hole and electron sides, respectively. Thus, it introduces an important electron-hole asymmetry. We see below that this plaquette quantum critical point results in a formation of a soft-fermion mode, i.e., a DOS peak at the Fermi energy when investigating it in a crystal of plaquettes. We argue that these soft fermions favor the formation of the

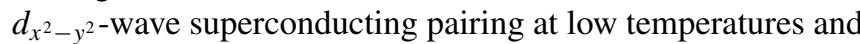
of the pseudogap at high temperatures. At smaller $t^{\prime}$ this critical point shifts to larger $U$, and at $t^{\prime} / t=0$ its coordinates are $U=4.58, \mu=0.72$. It is worthwhile to point out that at optimal values of $t^{\prime}$ antiferromagnetic order is suppressed due to frustrations. As soon as we add a fermionic bath to the plaquette within the spirit of CDMFT or density-matrix embedding theory (DMET) [38] with only four bath sites, a stable singlet solution forms with an almost equal mixture of all $N=2-4$ sectors, which again is favorable for the superconducting state as is shown below.

\section{PLAQUETTE IN THE BATH}

The appearance of the DOS peak at the Fermi energy leads to a universal instability in the sense that the susceptibility diverges in many different channels (magnetic, superconducting, charge-density wave, etc.). From the weak-coupling point of view this was discussed in the framework of the van Hove scenario of HTSC [39-41]. In order to study the interplay of different instability channels from the strong-coupling perspective we introduce a simple model of an embedded plaquette in the spirit of DMET [38]. To this aim we add to the plaquette's Hamiltonian a hybridization with four fermionic bath states, one bath state per corner of the plaquette, see Fig. 2(a). We use an exact diagonalization technique, namely, the Lanczos scheme with a Hilbert space size of $2^{16}$ 


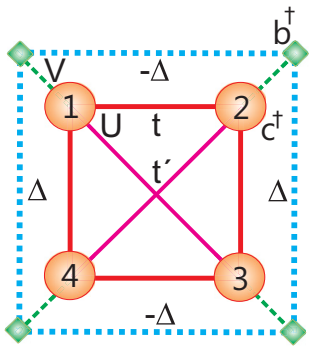

(a)

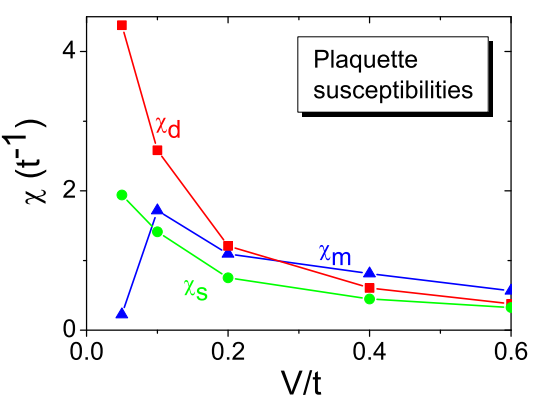

(b)
FIG. 2. (a) Sketch of the plaquette in the four-site bath with the

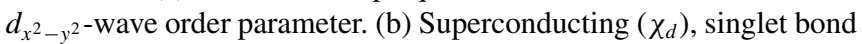
order $\left(\chi_{s}\right)$, and antiferromagnetic $\left(\chi_{m}\right)$ susceptibilities of the plaquette in a bath as a function of the hybridization $V$ for $U=3$ and $\mu=0.27$.

without any symmetry restrictions. Furthermore, we introduce different symmetry-breaking fields acting on the bath fermions $b_{i \sigma}^{\dagger}, b_{j \sigma}$, i.e., $d_{x^{2}-y^{2}}$-wave pairing, singlet magnetic states on the bonds, and the conventional Néel antiferromagnetic state,

$$
\begin{aligned}
h_{d} & =\sum_{\sigma=\uparrow, \downarrow, i=1 \ldots 4}(-1)^{i} \sigma \Delta_{d}\left(b_{i, \sigma} b_{i+1,-\sigma}+\text { H.c. }\right), \\
h_{s} & =\sum_{\sigma=\uparrow, \downarrow, i=1 \cdots 4}(-1)^{i} \sigma \Delta_{s}\left(b_{i, \sigma}^{\dagger} b_{i+1,-\sigma}+\text { H.c. }\right), \\
h_{m} & =\frac{1}{2} \sum_{\sigma=\uparrow, \downarrow, i=1 \cdots 4}(-1)^{i} \sigma \Delta_{m} b_{i, \sigma}^{\dagger} b_{i, \sigma} .
\end{aligned}
$$

Here we assume periodic boundary conditions, that means for $i=4$, we define $i+1=1$. We switch on small fields $\Delta_{d}=\Delta_{s}=\Delta_{m}=0.01 t$ simultaneously and calculate numerically their associated susceptibilities of the plaquette. The hybridization $V$ between fermions $c_{i \sigma}^{\dagger}$ and $b_{j \sigma}^{\dagger}$ breaks the sixfold degeneracy of the plaquette's quantum critical point, and without external fields it results in a singlet ground state, see Fig. 2(a). The $d_{x^{2}-y^{2}}$-wave superconducting [Eq. (3)] and the magnetic bond-singlet [Eq. (4)] external fields respect quantum entanglement of the singlet character of the ground state, whereas the Néel field Eq. (5) destroys it. Being classical in its nature, the Néel state is expected to be the most stable for sufficiently strong coupling with the environment $V$ [42] or high temperatures [43]. For an infinite system different types of order can be found by studying divergences of susceptibilities. Since in DMET we deal with finite systems, the susceptibilities remain finite up to zero temperature, and we assume that the largest susceptibility of the cluster, shown in Fig. 2(a), signals the corresponding order of the crystal. The computational results are shown in Fig. 2(b) as a function of the hybridization parameter $V$. One can see that the $d_{x^{2}-y^{2}}$-wave superconducting pairing always wins in comparison with the singlet bond pairing and is more favorable than the Néel order for $V \leqslant 0.2$. The self-consistent plaquette-Bethe DMFT for the cluster case (see below) with optimal HTSC parameters corresponds to $V=0.1$. The singlet ground state near the plaquette's quantum critical point favors $d_{x^{2}-y^{2}}$-wave superconductivity rather than magnetic ordering. This result

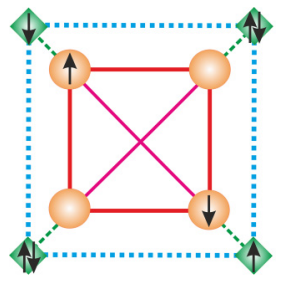

(a)

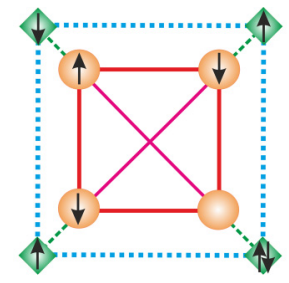

(b)

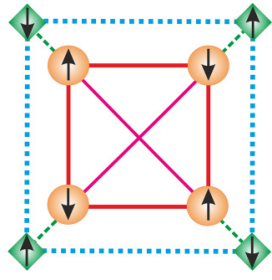

(c)
FIG. 3. The main contributions to the plaquette's singlet ground state in a four-site bath with the $d_{x^{2}-y^{2}}$-wave order parameter is the hybridization $V=0.2 \Delta_{d}=0.05$ for $U=3$ and $\mu=0.27$. (a) Sector $N=2$ with coefficient $=0.05$ and four antisymmetric contributions. (b) Sector $N=3$ with coefficient $=0.06$ and eight antisymmetric contributions. (c) Sector $N=4$ with coefficient $=0.08$ and two antisymmetric contributions.

agrees well with large-scale CDMFT calculations for optimal doping $[28,29]$.

In order to elucidate important properties of the plaquette singlet in the superconducting bath we visualize the main contributions to the ground-state singlet in Figs. 3(a)-3(c). There are three main contributions by the single-plaquette sectors with $N=2-4$ electrons, which have almost equal spectral weights. In particular, it means a strong mixture of states that differ by two electrons, i.e., by a local Cooper pair on the bond. This is understood as an important detail for superconductivity since the degenerate quantum critical point (circle in Fig. 1) consists of states with the same total number of electrons $\left(N_{\text {tot }}=8\right)$, but none of those show a double occupation on the plaquette. It occurs only on bath sites that have no Hubbard interaction, see Figs. 3(a)-3(c). The degeneracy of the quantum critical point splits into four states for $N=2$, eight states for $N=3$, and two states for $N=4$. Taking into account the total number of these states and including all their antisymmetric singlet combinations, we observe that $85 \%$ of the ground-state components are related to the quantum critical point. This means that the system as a whole perfectly screens the strong Coulomb interactions (Hubbard $U$ is about $70 \%$ of the bandwidth), and the plaquette states with $N=2-4$ electrons contribute equally to the ground-state singlet of the total system. The fact that there are no double occupied states inside the plaquette and only on the bath sites with no Hubbard interactions was expected to occur only in the limit $U \gg W$, obtained by the perfect Gutzwiller projector. The crucial role of the Gutzwiller projector for the physics of high- $T_{c}$ superconductivity was emphasized by Anderson [12]. We see that the situation is much more subtle: A specific symmetry of the ground state at the plaquette's quantum critical point increases effectively the single-site $U$ by suppressing double occupations on plaquette sites. But, at the same time, it decreases the effective $U$ for the plaquette in a sense that one does not have to pay an additional energy for adding two more electrons to the plaquette as a whole. This situation is very nontrivial; it demonstrates clearly that discussions of strong correlations for the high- $T_{c}$ cuprates would be based on a rather four-orbital Hubbard model, corresponding to a lattice built from plaquettes, than on an initial single-band Hubbard model for copper sites. 


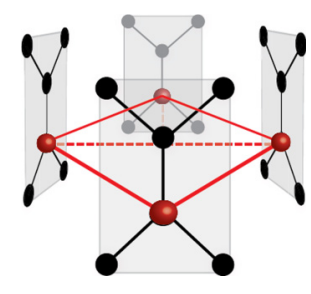

(a)

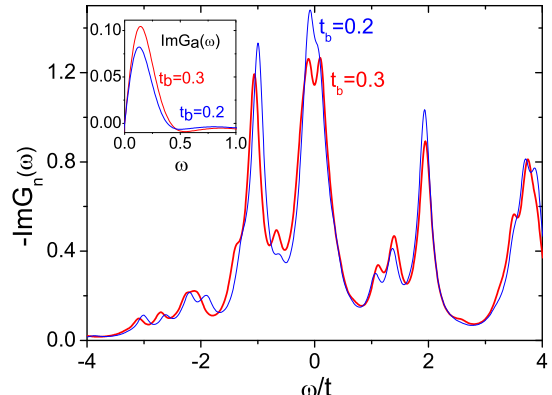

(b)
FIG. 4. (a) Sketch of the plaquette-Bethe lattice with connectivity $q=2$. Only one plaquette is shown for simplicity. (b) Local part of the normal Green's function of the $d_{x^{2}-y^{2}}$-wave solution in the plaquette-Bethe lattice near optimal values of $t_{b}$ for $U=3$ and $n=0.85$ and (the inset) the nonlocal part of the anomalous Green's function.

\section{PLAQUETTE-BETHE LATTICE}

As a next step towards a more realistic description of the cuprate crystal, we consider a plaquette-Bethe model with all sites arranged in quadrupole Bethe "planes" and interconnected in a plaquettelike manner, see Fig. 4(a). The plaquette CDMFT becomes exact for this model when the connectivity of the Bethe sublattice $q$ tends to infinity. We obtain similar results as for the double-Bethe model for a two-site cluster $[42,44]$. The bath Green's function in Nambu representation for this model reads

$$
\hat{\mathcal{G}}^{-1}(i \omega)=i \omega \mathbb{1}+\left(\mu-\hat{h}_{0}\right) \sigma_{z}-t_{b} \sigma_{z} \hat{G}(i \omega) \sigma_{z} t_{b},
$$

where $\sigma_{z}$ is the Pauli matrix and including site degrees of freedom $\hat{G}(i \omega)$ is an $8 \times 8$ matrix of the superconducting Green's function for the plaquette in the bath. We discretize the bath Green's function with only four states similar to the DMET approach using the Lanczos scheme in order to find the matrix Green's function of the superconducting states [28,29]. As mentioned above, there is a sixfold degenerate ground state for $t_{b}=0$ at the quantum critical point. At sufficiently small hybridizations, i.e., small $t_{b}$ in the plaquette-Bethe model, the system becomes metallic with a slightly broadened peak in the DOS at the Fermi energy. The ground state is $d_{x^{2}-y^{2}}$-wave superconducting at low temperatures $[28,29]$. However, when $t_{b}$ increases a quantum phase transition occurs with the destruction of the singlet states and a formation of the energy gap in the single-electron excitation spectrum. The latter can be observed in the normal part of the one-electron Green's function at $t_{b}=0.3$, see Fig. 4(b). The energy gap of the states can be estimated at $0.2 t$, that is, an order of magnitude larger than the superconducting gap. For $t=0.25 \mathrm{eV}$ [13] this results in a pseudogap on the order of $50 \mathrm{meV}$, which is observed experimentally [45]. In regard to the double-Bethe model this corresponds to a transition of a quantum entangled singlet state to a classical Néel state [42]. Importantly, the anomalous (superconducting) part of the Green's function has a maximum exactly at this transition, see the right inset in Fig. 4(b). Similar behavior has been observed recently experimentally for the maximum of the superconducting order parameter at the localized-delocalized transition point in the strongly correlated

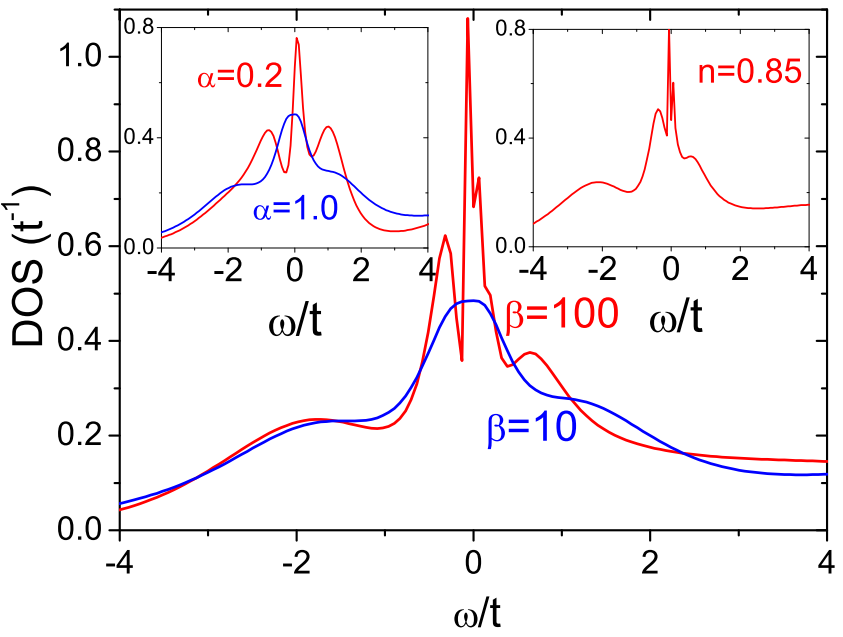

FIG. 5. Density of states for both spins of the plaquette CDMFT for $U=6$ and $\mu=0.54$ for different temperatures, (the left inset) with plaquette-lattice hoppings scaled by a factor of $\alpha$ at $\beta=10$ and (the right inset) for optimal doping $n=0.85$ at $\beta=100$.

organic superconductors [46] as well as for the BCS-BEC crossover in cold-atom systems [47].

\section{PLAQUETTE CDMFT}

Finally, we perform the standard CDMFT calculations using a strong-coupling continuous-time quantum Monte Carlo solver [48,49] in the normal state. Since a transition to the periodic plaquette in the crystal increases the bandwidth by a factor of 2 due to doubling of the coordination numbers compared to the isolated plaquette, we increase the values of $U$ and $\mu$ by the factor of 2 . Note, that the natural energy unit is the bandwidth $W$ rather than the hopping $t$. Furthermore, we use the value of $U / t=6$, which approximately describes real cuprate materials [50].

The calculated local DOS obtained by maximum-entropy analytic continuation [51] is shown in Fig. 5. We observe that for sufficiently high temperatures there is a broad peak at the Fermi energy originating from the plaquette quantum critical point. This relation is illustrated in the inset of Fig. 5. We artificially scaled the hopping between plaquettes by a factor of $\alpha$ ranging from 0 to the physical value 1 .

At lower temperatures a pseudogap is formed. It is well known in all HTSC materials and considered as one of its most striking features [45]. Sometimes this pseudogap is also considered to be the precursor of the superconducting gap (formation of incoherent Cooper pairs above $T_{c}$ ) or as a smeared antiferromagnetic gap (shadow bands) [45]. However, both interpretations have problems when they get compared to experiments [45]. Within our scheme it is natural to interpret this pseudogap as a pseudohybridization gap similar to that arising in Kondo lattices [52] or intermediate valence semiconductors [53]. From this point of view the pseudogap in HTSC materials originates from the Fano antiresonance due to embedding of the soft-fermion mode of the plaquette (discussed above) into a continuous band spectrum of the lattice. Indeed, from the degeneracy between the states with $N=3$ and $N=3 \pm 1=2,4$ of the isolated plaquette 
immediately follows by the Lehmann spectral representation that the atomic Green's function of the plaquette has a pole at $E=0$. In a lattice built from plaquettes this pole is mixed by hopping with the other spectral components of the Green's function, which otherwise would form a regular strongly correlated band. However, the mixture with the $E=0$ peak leads to a singularity in the self-energy and thus to the gap opening. The broadening of the $E=0$ peak transforms the gap into the pseudogap. A similar effect is produced by the Kondo peak in the periodic Anderson model [52] but with the important difference that the spectral weight of the Kondo peak is proportional to the Kondo temperature and therefore small, whereas in the model discussed here the soft fermion has a noticeable spectral weight. It leads to a much larger energy scale of the pseudogap compared to the Kondo lattices. The singularity in the self-energy and the gap opening in systems with flat bands has been discussed recently from the point of view of phenomenological Fermi-liquid theory [54].

The role of soft-fermion modes, the hidden fermion, has been discussed in Refs. [55-57]. However, it was not done in the context of the quantum critical point of the plaquette. This relation is the main message of our paper.

The density of states for optimal doping $n=0.85$ in the self-consistent CDMFT calculations is shown in the right inset of Fig. 4(b). Its chemical potential converges to a value of $\mu=1.2$, which is very similar to that of the fixed $\mu$ of the quantum critical point in the isolated plaquette. Furthermore, we have calculated a low-temperature superconducting state with CDMFT, a Lanczos solver, eight-bath sites, and with a CTQMC cluster solver and found similar results to many other calculations [17-35].

\section{CONCLUSIONS}

To conclude, we developed a picture of HTSC based on the existence of a quantum critical point at the crossing of the ground-state energies in the $N=2-4$ sectors within the plaquette for parameters close to the optimal doping, $t^{\prime}$ being of crucial importance. Contrary to the original RVB theory of Anderson [6], we start with the local valence-bond formation in the doped plaquette. The difference can be illustrated by comparison with the exactly solvable one-dimensional quantum spin model [36]. The prototype state for the RVB is the Bethe ansatz solution for the antiferromagnetic $S=1 / 2$ Heisenberg model in the nearest-neighbor approximation. For the model with first- and second-nearest-neighbor interactions $J_{2} / J_{1}=1 / 2$, the ground state is known exactly too. It can be represented as a product state of local valence-bond singlets [36]. For the two-dimensional $J_{1}, J_{2}$ model [58,59] recent calculations show the formation of a plaquette valence-bond state with a tendency towards the $d$-wave superconductivity for the $t-J_{1}, J_{2}$ model [60]. The second-nearest-neighbor hopping $t^{\prime}$ seems to play a similar role in the Hubbard model. The optimal superconducting region is related to a localizeddelocalized transition of plaquette valence-bond states in the plaquette. It would be interesting to describe the formation of global singlet states with plaquette valence-bond states using the matrix product scheme [61] since the CDMFT scheme breaks translational symmetry.

Formation of the soft-fermion mode near the optimal doping has an analog in the weak-coupling theory within the van Hove scenario of HTSC [62]. Due to the formation of flat bands of many-body origin $[62,63]$ there is a whole region of parameters $t^{\prime}, U, \mu$ in that the Fermi-liquid description is broken. However, we believe that the strong-coupling description presented here is more relevant for real HTSC materials, which are characterized by large values of $U$ [50].

\section{ACKNOWLEDGMENTS}

We thank B. L. Altshuler, O. K. Andersen, P. W. Anderson, A. Georges, A. Millis, M. Potthoff, T. Wehling, and J. Wiebe for helpful discussions as well as T. Berberich and M. Valentyuk for their assistance with the figures, L. Boehnke for the MAXENT code, and S. Okamoto for the LANCZOS-SC code. A.I.L. acknowledges support from the DFG Grant No. SFB-668 and The Hamburg Centre for Ultrafast Imaging, M.I.K. acknowledges financial support from ERC (Project No. 338957 FEMTO/NANO) and from NWO via the Spinoza Prize. Computations have been performed at the NIC, Forschungszentrum Jülich, under Project No. HHH26.
[1] J. G. Bednorz and K. A. Müller, Z. Phys. B: Condens. Matter 64, 189 (1986).

[2] A. Damascelli, Z. Hussain, and Z.-X. Shen, Rev. Mod. Phys. 75, 473 (2003).

[3] J. Orenstein, and A. J. Millis, Science 288, 468 (2000).

[4] M. H. Hamidian, S. D. Edkins, C. K. Kim, J. C. Davis, A. P. Mackenzie, H. Eisaki, S. Uchida, M. J. Lawler, E.-A. Kim, S. Sachdev, and K. Fujita, Nat. Phys. 12, 150 (2016).

[5] P. W. Anderson, Adv. Phys. 46, 3 (1997).

[6] P. W. Anderson, The Theory of Superconductivity in the High- $T_{c}$ Cuprates (Princeton University Press, Princeton, 1997).

[7] D. J. Scalapino, Rev. Mod. Phys. 84, 1383 (2012).

[8] J. Schmalian, D. Pines, and B. Stojkovic, Phys. Rev. Lett. 80, 3839 (1998).
[9] M. Imada, A. Fujimori, and Y. Tokura, Rev. Mod. Phys. 70, 1039 (1998).

[10] E. Dagotto, Rev. Mod. Phys. 66, 763 (1994).

[11] H. J. A. Molegraaf, C. Presura, D. van der Marel, P. H. Kes, and M. Li, Science 295, 2239 (2002).

[12] P. W. Anderson, Int. J. Mod. Phys. B 25, 1 (2011).

[13] O. K. Andersen, A. I. Liechtenstein, O. Jepsen, and F. Paulsen, J. Phys. Chem. Solids 56, 1573 (1995).

[14] E. Pavarini, I. Dasgupta, T. Saha-Dasgupta, O. Jepsen, and O. K. Andersen, Phys. Rev. Lett. 87, 047003 (2001).

[15] A. Georges, G. Kotliar, W. Krauth, and M. J. Rozenberg, Rev. Mod. Phys. 68, 13 (1996).

[16] A. I. Lichtenstein and M. I. Katsnelson, Phys. Rev. B 62, R9283(R) (2000). 
[17] T. Maier, M. Jarrell, T. Pruschke, and M. H. Hettler, Rev. Mod. Phys. 77, 1027 (2005).

[18] T. A. Maier, M. S. Jarrell, and D. J. Scalapino, Phys. Rev. Lett. 96, 047005 (2006).

[19] K. Haule and G. Kotliar, Phys. Rev. B 76, 104509 (2007).

[20] M. Ferrero, P. S. Cornaglia, L. De Leo, O. Parcollet, G. Kotliar, and A. Georges, Europhys. Lett. 85, 57009 (2009).

[21] M. Ferrero, P. S. Cornaglia, L. De Leo, O. Parcollet, G. Kotliar, and A. Georges, Phys. Rev. B 80, 064501 (2009).

[22] E. Gull, M. Ferrero, O. Parcollet, A. Georges, and A. J. Millis, Phys. Rev. B 82, 155101 (2010).

[23] E. Khatami, K. Mikelsons, D. Galanakis, A. Macridin, J. Moreno, R. T. Scalettar, and M. Jarrell, Phys. Rev. B 81, 201101(R) (2010).

[24] E. Gull, O. Parcollet, and A. J. Millis, Phys. Rev. Lett. 110, 216405 (2013).

[25] D. Senechal, P.-L. Lavertu, M.-A. Marois, and A.-M. S. Tremblay, Phys. Rev. Lett. 94, 156404 (2005).

[26] M. Aichhorn, E. Arrigoni, M. Potthoff, and W. Hanke, Phys. Rev. B 76, 224509 (2007).

[27] E. Gull, P. Werner, X. Wang, M. Troyer, and A. J. Millis, Europhys. Lett. 84, 37009 (2008).

[28] M. Civelli, Phys. Rev. Lett. 103, 136402 (2009).

[29] S. S. Kancharla, B. Kyung, D. Senechal, M. Civelli, M. Capone, G. Kotliar, and A.-M. S. Tremblay, Phys. Rev. B 77, 184516 (2008).

[30] S. Okamoto and T. A. Maier, Phys. Rev. Lett. 101, 156401 (2008).

[31] G. Sordi, K. Haule, and A.-M. S. Tremblay, Phys. Rev. B 84, 075161 (2011).

[32] S. Okamoto, D. Sénéchal, M. Civelli, and A.-M. S. Tremblay, Phys. Rev. B 82, 180511 (2010).

[33] G. Sordi, P. Sémon, K. Haule, and A.-M. S. Tremblay, Sci. Rep. 2, 547 (2012).

[34] X. Chen, J. P. F. LeBlanc, and E. Gull, Phys. Rev. Lett. 115, 116402 (2015).

[35] L. Fratino, P. Sémon, G. Sordi, and A.-M. S. Tremblay, Sci. Rep. 6, 22715 (2016).

[36] I. Affleck, J. Phys.: Condens. Matter 2, 405 (1990).

[37] H. Yao, W.-F. Tsai, and S. A. Kivelson, Phys. Rev. B 76, 161104(R) (2007).

[38] B.-X. Zheng and G. K.-L. Chan, Phys. Rev. B 93, 035126 (2016).

[39] V. Y. Irkhin, A. A. Katanin, and M. I. Katsnelson, Phys. Rev. B 64, 165107 (2001).

[40] A. P. Kampf and A. A. Katanin, Phys. Rev. B 67, 125104 (2003).

[41] W. Metzner, M. Salmhofer, C. Honerkamp, V. Meden, and K. Schönhammer, Rev. Mod. Phys. 84, 299 (2012).
[42] H. Hafermann, M. I. Katsnelson, and A. I. Lichtenstein, Europhys. Lett. 85, 37006 (2009).

[43] J. Otsuki, H. Hafermann, and A. I. Lichtenstein, Phys. Rev. B 90, 235132 (2014).

[44] G. Moeller, V. Dobrosavljevic, and A. E. Ruckenstein, Phys. Rev. B 59, 6846 (1999).

[45] M. Hashimoto, I. M. Vishik, R.-H. He, T. P. Devereaux, and Z.-X. Shen, Nat. Phys. 10, 483 (2014).

[46] R. H. Zadik, Y. Takabayashi, G. Klupp, R. H. Colman, A. Y Ganin, A. Potočnik, P. Jeglič, D. Arčon, P. Matus, K. Kamarás, Y. Kasahara, Y. Iwasa, A. N. Fitch, Y. Ohishi, G. Garbarino, K. Kato, M. J. Rosseinsky, and K. Prassides, Sci. Adv. 1, e1500059 (2015).

[47] C. A. Regal, M. Greiner, and D. S. Jin, Phys. Rev. Lett. 92 040403 (2004).

[48] E. Gull, A. J. Millis, A. I. Lichtenstein, A. N. Rubtsov, M. Troyer, and P. Werner, Rev. Mod. Phys. 83, 349 (2011).

[49] P. Seth, I. Krivenko, M. Ferrero, and O. Parcollet, Comput. Phys. Commun. 200, 274 (2016).

[50] B. Dalla Piazza, M. Mourigal, M. Guarise, H. Berger, T. Schmitt, K. J. Zhou, M. Grioni, and H. M. Ronnow, Phys. Rev. B 85, 100508(R) (2012).

[51] M. Jarrell and J. E. Gubernatis, Phys. Rep. 269, 133 (1996).

[52] R. Bulla, T. A. Costi, and T. Pruschke, Rev. Mod. Phys. 80, 395 (2008).

[53] V. Y. Irkhin and M. I. Katsnelson, Zh. Eksp. Teor. Fiz. 90, 1080 (1986) [Sov. Phys. JETP 63, 631 (1986)].

[54] V. A. Khodel, Pis'ma Zh. Eksp. Teor. Fiz. 103, 795 (2016).

[55] S. Sakai, M. Civelli, and M. Imada, Phys. Rev. Lett. 116, 057003 (2016).

[56] S. Sakai, Y. Motome, and M. Imada, Phys. Rev. B 82, 134505 (2010).

[57] S. Sakai, S. Blanc, M. Civelli, Y. Gallais, M. Cazayous, M.A. Méasson, J. S. Wen, Z. J. Xu, G. D. Gu, G. Sangiovanni, Y. Motome, K. Held, A. Sacuto, A. Georges, and M. Imada, Phys. Rev. Lett. 111, 107001 (2013).

[58] L. Capriotti and S. Sorella, Phys. Rev. Lett. 84, 3173 (2000).

[59] S.-S. Gong, W. Zhu, D. N. Sheng, O. I. Motrunich, and M. P. A. Fisher, Phys. Rev. Lett. 113, 027201 (2014).

[60] D. Poilblanc, P. Corboz, N. Schuch, and J. I. Cirac, Phys. Rev. B 89, 241106(R) (2014).

[61] F. Verstraete, J. I. Cirac, and V. Murg, Adv. Phys. 57, 143 (2008).

[62] V. Y. Irkhin, A. A. Katanin, and M. I. Katsnelson, Phys. Rev. Lett. 89, 076401 (2002).

[63] D. Yudin, D. Hirschmeier, H. Hafermann, O. Eriksson, A. I Lichtenstein, and M. I. Katsnelson, Phys. Rev. Lett. 112, 070403 (2014). 